\title{
Impact of COVID-19 on Heart Failure Hospitalizations
}

\author{
Savalan Babapoor-Farrokhran ${ }^{1}$ (D) Jafar Alzubi ${ }^{1} \cdot$ Zachary Port $^{1} \cdot$ Naveen Sooknanan $^{2} \cdot$ Zaid Ammari $^{1}$. \\ Mohammad Al-Sarie ${ }^{1} \cdot$ Behnam Bozorgnia ${ }^{1,3}$
}

Accepted: 13 June 2021 / Published online: 25 June 2021

(C) The Author(s), under exclusive licence to Springer Nature Switzerland AG 2021

\begin{abstract}
The Novel Coronavirus Disease 2019 (COVID-19) pandemic has transformed individual lives and societal framework on a global scale, and in no other sector is this more evident than healthcare. Herein, we aim to describe the impact of the current COVID-19 pandemic and its associated restrictions on heart failure (HF) admissions. In this retrospective cohort study, we obtained administrative data for patients with a primary discharge diagnosis of HF (identified by ICD-10 code) with discharge dates ranging from January 2019 to November 2020. The study is comprised of 2 distinct sub-cohorts: HF admissions during the COVID-19 pandemic (case) period from March 2020 to October 2020 and corresponding control period during the previous year (March 2019 to December 2019). Primary outcome analysis involved comparison of total and daily HF admissions and secondary outcomes included hospital Length of Stay (LOS) and 30-day readmissions. The number of total HF admissions and average daily admissions were significantly lower in 2020 compared to 2019 (774 vs. 864; $p<0.001$ and 3.17 vs. 3.53 days; $p<0.001$ ), respectively. Average length of stay was significantly higher between March and October 2020 relative to the same months in 2019 (6.05 vs. 5.25 days; $p$ < 0.001). Thirty-day readmission rates were also significantly higher in March-October 2020 compared to the same months in 2019 (20.6\% vs. $19.1 \%$; < 0.001). During the pandemic, both readmission rates and length of stay for HF-related admissions were significantly impacted. The COVID-19 pandemic significantly impacted HF-related admissions as well as associated LOS and 30-day readmissions. High-risk patients should be identified carefully, and timely and appropriate treatment should be provided.
\end{abstract}

Keywords Heart failure $\cdot$ COVID-19 $\cdot$ Heart failure readmission $\cdot$ Heart failure length of stay

\section{Introduction}

The Novel Coronavirus Disease 2019 (COVID-19) quickly developed into the largest global public health crisis in recent times. Thought to have originated in Wuhan, China, in late 2019 due to initial bat-to-human transmission, the virus

Savalan Babapoor-Farrokhran and Jafar Alzubi contributed equally to this work.

This article is part of the Topical Collection on COVID-19

Savalan Babapoor-Farrokhran

Babapoos@einstein.edu

1 Department of Medicine, Division of Cardiology, Einstein Medical Center, 5501 Old York Road, Philadelphia, PA 19141, USA

2 Department of Medicine, Einstein Medical Center, Philadelphia, PA 19141, USA

3 Sidney Kimmel Medical College, Thomas Jefferson University, Philadelphia, PA 19107, USA quickly reached pandemic status, reaching epidemic status in early 2020 [1]. By March 2020, many countries began to issue major public health restrictions such as quarantines and mask mandates in an attempt to control viral spread [2]. Another such measure carried out by many major hospitals in the USA was decreasing the number of elective medical procedures performed during the pandemic, as well as limiting outpatient visits to either a Telemedicine format or to patients who needed immediate medical attention [3]. During the pandemic, the primary focus of many hospitals shifted, out of necessity, from a wide breadth of medical care to treating the morbidity and mortality of COVID-19 patients, with multiple studies demonstrating this phenomenon with decreased inpatient admissions for non-COVID-19-related problems, including asthma, atrial fibrillation, acute Coronary Syndrome (ACS), and influenza [3-7]. The COVID-19 pandemic has been shown to similarly impact hospital admissions for heart failure (HF), with the most robust data originating from major tertiary centers in Europe and the UK [7, 8]. Data on the COVID-19 pandemic's impact on US HF admissions remains 
limited to observational data from Mississippi and Tennessee, with a particular lack of data from the Northeast USA, which was hit disproportionately hard during the first wave of the pandemic $[9,10]$. This study aims to demonstrate the impact of the COVID-19 pandemic on heart failure admissions in a major urban health center in North Philadelphia.

\section{Methods}

We performed a retrospective cohort study analyzing HF admissions in a single urban hospital in North Philadelphia throughout a study period between January 2019 and November 2020. Admission data was collected by obtaining administrative data from the hospital for patients with a primary discharge diagnosis of HF, identified by ICD-10 code, with discharge dates falling within the prespecified study period as outlined above. HF admission data was then split into two distinct time periods based on the time period correlating to the COVID-19 pandemic in the USA as well as the administrative data regarding active inpatient COVID-19 cases during these time periods. The control, or non-COVID, period inclusively ranged from March 2019 to October 2019 while the case, or COVID, period inclusively ranged from March 2020 to March 2020, with an inter-period duration of approximately 5 months to ensure equal duration of the two periods as well as lack of clarity regarding the exact start of the pandemic locally. The primary outcome measured in this study was HF admissions between the two periods, while secondary outcomes included total hospital length of stay (LOS) and 30-day readmission rates during these time periods. Where applicable, sample means were calculated for continuous data, which were compared using a two-sample T-test, and P-values less than 0.05 were found to be statistically significant. Statistical analysis was performed using SPSS.

\section{Results}

Through the inclusion criteria outlined above, a total of 1638 patients were included in this study, 864 (53\%) in the nonCOVID group and $774(47 \%)$ in the COVID group. The primary outcome of total HF admissions and daily HF admissions was significantly lower in the case period compared to the control period (774 vs. $864 ; p<0.001$ and 3.17 vs. 3.53 days; $\mathrm{p}<0.001$, respectively) (Fig. 1A, B; Table 1). Within the case period, total HF admissions were lowest in April 2020 ( $\mathrm{n}=59$ ), which corresponded to the month in which the number of COVID-positive admissions were highest ( $\mathrm{n}$ = 423) (Fig. 1A). Furthermore, total HF admissions were lower during the beginning of the case period, specifically through March to June 2020 compared to the same months in the control period (Fig. 1A, B). Similarly, in months of the case period corresponding to relatively lower numbers of COVIDpositive admissions, differences in total and daily HF admissions between the two periods, while still statistically significant, were less pronounced in magnitude compared to months with high rates of COVID-positive admissions (Fig. 1A, G).

Regarding secondary outcome analysis, average hospital LOS was significantly longer in the case period compared to the control period (6.05 vs. 5.25 days; $p<0.001$ ) (Fig. 1C, D; Table 1). Thirty-day readmission rates were also significantly higher in in the case period $(20.6 \%$ vs. $19.1 \%$; $p<0.001)$ (Fig. 1E, F; Table 1). The number of HF admissions who tested positive for COVID was negligible (Fig. 1G).

\section{Discussion}

The COVID-19 pandemic has created an unprecedented global health pandemic which has been implicated in the loss of over 1 million lives worldwide by the end of 2020 [1, 2]. Due a justified focus on the morbidity and mortality associated with the virus itself, many other chronic medical conditions and their associated health implications were given less attention. From an infectious disease standpoint, the increased focus on public health measures such as hand hygiene, social distancing, and proper face and eye protection have understandably led to a reduction in other notable communicable diseases, such as influenza and pneumococcal disease [6, 11]. Perhaps more interesting is the current data suggesting a trend towards reduced hospital admissions for diagnoses other than COVID-19, including other cardiac conditions such as ACS and new-onset atrial fibrillation [3, 5]. Early studies from Europe and the UK have described reductions in HF admission and readmissions during the COVID-19 pandemic $[3,8$, 12-15]. Currently, robust prospective and retrospective data regarding the impact of the COVID-19 pandemic on HF admission in the USA remain limited. Retrospective data in the forms of Editorials, in which patient selection and data collection were performed through either admission or discharge diagnoses, as well as one Letter to the Editor, sum up the current US data pool on the topic $[9,10,16]$.

In one such study, Hall et al. examined HF-related hospitalization trends between January and April 2020, compared to a similar period in 2019 , in a large, tertiary academic medical center in Mississippi; patients were selected using electronic health record (EHR) and hospital discharge databases based on ICD-10 codes [9]. Despite week-to-week variability, a statistically significant trend towards reduced HF-related hospitalizations occurred in March and April 2020 regardless of heart failure subtype (reduced, preserved, or mid-range ejection fraction). This decline in HF admissions also coincided with the pandemic reaching emergency status in the state, with a declaration of emergency in Mississippi on March 14, 
A

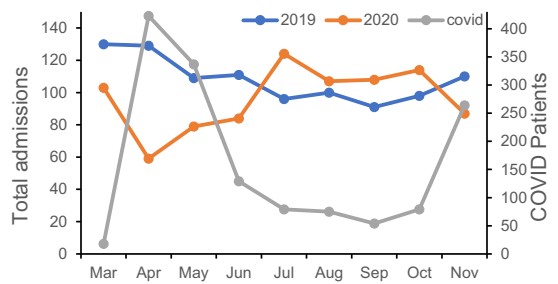

C

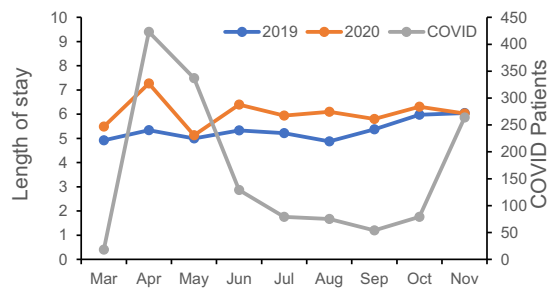

E

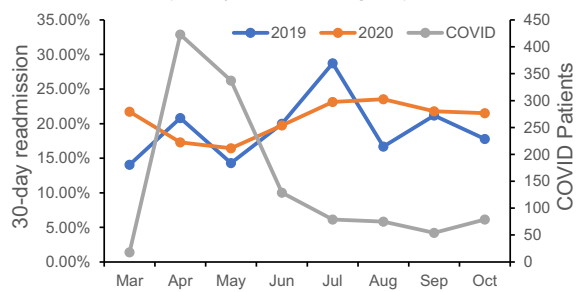

Fig. 1 Number of heart failure admissions, length of stay, 30-day readmission, and number of COVID-19 admissions. A The number of total admissions during March-November 2019 and 2020. Gray line indicates the number of COVID-19 admissions from March-November 2019. B Representative graph demonstrating total admissions in 2019 and 2020. C Average length of stay for HF admissions during MarchNovember 2019 and 2020. Gray line indicates the number of COVID-

2020 [9]. Further data from an accepted manuscript from Cox, Lai, and Lindenfield noted a decline in HF hospitalization of $62 \% \pm 7 \%$ between March 22nd and April 20th, 2020, compared to the same calendar days the previous year $(11 \% \pm 12$ $\%$ vs $-46 \% \pm 16 \%, \mathrm{p}=0.0012$ ) [10]. This single center study was conducted at a major academic medical center in Nashville, Tennessee, with patients being recruited according to a validated algorithm including factors such as ICD-10 code diagnosis, basic natriuretic peptide (BNP) level, and administration of IV diuretic therapy [17]. The study also noted similar admission systolic blood pressure, sodium and creatinine levels, and patient ages, between the two time periods [10]. Finally, Barghash and Pinney describe a dramatic reduction in HF-related admissions in metropolitan New York in the form of a Letter to the Editor, particularly coinciding with the mandatory stay-at-home mandate for non-essential workers issued on March 22nd, 2020 [16]. Unfortunately, exact data
B



19 admissions from March-November 2019. D Representative graph demonstrating average length of stay in 2019 and 2020. $\mathbf{E}$ Representative graph demonstrating 30-day readmission in 2019 and 2020. F 30-day readmission for HF patients during March-November 2019 and 2020. Gray line indicates the number of COVID-19 admissions from March-November 2019. G Number of HF who were admitted with COVID-19. *** p-value $<0.001$

regarding the magnitude of decline in HF admissions was not provided given the nature of the publication, limiting its clinical utility.

In line with current data, our study redemonstrates a significant decline in HF-related hospitalizations during the period correlating to the COVID-19 pandemic, with the lower daily admission rates occurring during periods where the COVID19 inpatient census was the highest. However, unlike data obtained by Cox, Lai, and Lindenfield, hospital LOS was noted to be significant higher during the COVID-19 pandemic period in our study, suggesting larger retrospective data will be needed to clarify this discrepancy. Thirty-day readmission rates, which were not commented upon by the above studies, were significantly higher in our study. The increase in LOS for HF patients can be explained by a delay in routine cardiac care and the late presentation of many patients, as previously postulated by the studies above $[9,10,16]$. This is partially

Table 1 Number of total admissions, average daily admission, average length of stay, and readmissions from March to October in 2019 and 2020

\begin{tabular}{llll}
\hline & March-October 2020 & March-October 2019 & p-value \\
\hline Total admission (n) & 774 & 864 & $<0.001$ \\
Daily admissions & $3.17(2.61-3.74)$ & $3.53(3.11-3.94)$ & $<0.001$ \\
LOS (days) & $6.05(5.52-6.59)$ & $5.25(4.96-5.55)$ & $<0.001$ \\
Readmissions (\%) & $20.6(18.5-22.8)$ & $19.1(15.2-0.23)$ & \\
\hline
\end{tabular}


the result of implementing certain hospital policies to decrease the potential risk of inadvertent exposure to the virus and maintain hospital capacity to keep up with expected COVID-19 admission surges, such as limiting inpatient studies such as echocardiograms, canceling elective outpatient procedures, and performing COVID swabs on all admitted patients. As stated by Hall et al. as well as Cox, Lai, and Lindenfield, registry and ICD-10 code-based patient enrollment is particularly difficult in HF patient where the diagnoses are primary clinical and may not be immediately apparent on hospital admission $[9,17]$. This is a limitation shared by our study and may be exacerbated during the COVID-19 pandemic by shared respiratory complains in patients with COVID-19 pneumonia, as well as policies in place in certain institutions to perform COVID-19 PCR testing on all admitted patients. Notably, the 30-day readmission rate during the study period was higher compared with the prior year. This suggests that the pandemic impacted the level of the care delivered during HF admissions as well as the outpatient care following the hospitalization of those patients. One possible explanation from an inpatient standpoint was an ongoing pressure to maintain high patient turnover and rapid discharges to ensure availability to the ever-growing number of COVID-19 hospitalizations. This was compounded by the need for airborne and respiratory isolation precautions in confirmed and suspected COVID-19 patients, eliminating the possibility of them being admitted to double-rooms; this created significant barriers from an administrative perspective, and significantly lowered the total available bed count of the hospital. From an outpatient perspective, many patients choose to have telehealth follow-ups instead of traditional in-person visits. Given that heart failure is a clinical diagnosis driven by the need for accurate volume assessment, telehealth visits by their very nature limit the capacity for accurate clinical assessments and may hinder the ability to adjust diuretic therapy in an effective manner. Finally, the decrease in rate of $\mathrm{HF}$ admissions during the pandemic may be attributable to apprehension of seeking medical attention during the national lockdown, a finding that has been hypothesized in similar studies $[9,10,16]$.

This study has inherent limitations related to its observational nature and relatively small sample size. In addition to the accuracy issues outlined above, the registry-based patient collection method based on ICD-10 code limits further data acquisition and extrapolation, including the use of important clinical biomarkers such as BNP, as well as information regarding the severity illness (e.g., NYHA Classification of symptoms on arrival) and cardiovascular and overall mortality. Nonetheless, the findings raise awareness of the importance of securing timely and appropriate treatment of all diseases during a pandemic, and demonstrate the need for further retrospective and prospective data to investigate and rectify these trends.

\section{Conclusion}

The COVID-19 pandemic has profoundly impacted the US healthcare system in a number of ways, including a necessitybased focus on major health complications related to the virus itself. As such, perhaps due to this excessive focus and partially from a hesitance of the general public to receive routine medical care, hospital admissions for reasons outside of COVID-19 pneumonia decreased dramatically, including cardiovascular diseases such as heart failure. Our study confirms a significant drop in HF admissions during the COVID-19 pandemic, in line with limited retrospective data from other regions in the USA, while also showing prolonged LOS and 30-day readmission rates leading to concern about the level of inpatient care and quality of subsequent outpatient follow-up. It will be important for more robust retrospective data to clarify these associations to ensure quality of cardiovascular care is not compromised during these unprecedented times.

Author Contribution SB and JA: equal contribution to conception and design, acquisition of the data or analysis, interpretation of the data, and writing the manuscript. ZA: drafting the article or revising it critically for important intellectual content. ZP: drafting the article or revising it critically for important intellectual content. NS: drafting the article or revising it critically for important intellectual content. MA: drafting the article or revising it critically for important intellectual content; BB: revising the article critically for important intellectual content.

Data Availability Not applicable.

Code Availability Not applicable.

\section{Declarations}

Ethics Approval Not applicable.

Consent to participate Not applicable.

Consent for Publication Not applicable.

Conflict of Interest The authors declare no competing interests.

\section{References}

1. Khan M, Adil SF, Alkhathlan HZ, Tahir MN, Saif S, Khan M, et al. COVID-19: a global challenge with old history, epidemiology and progress so far. Molecules. 2020;26(1).

2. Shereen MA, Khan S, Kazmi A, Bashir N, Siddique R. COVID-19 infection: Origin, transmission, and characteristics of human coronaviruses. J Adv Res. 2020;24:91-8.

3. De Filippo O, D'Ascenzo F, Angelini F, Bocchino PP, Conrotto F, Saglietto A, et al. Reduced rate of hospital admissions for ACS during Covid-19 outbreak in Northern Italy. N Engl J Med. 2020;383(1):88-9.

4. Grasselli G, Pesenti A, Cecconi M. Critical care utilization for the COVID-19 outbreak in Lombardy, Italy: early experience and forecast during an emergency response. Jama. 2020;323(16):1545-6. 
5. Holt A, Gislason GH, Schou M, Zareini B, Biering-Sørensen T, Phelps M, et al. New-onset atrial fibrillation: incidence, characteristics, and related events following a national COVID-19 lockdown of 5.6 million people. Eur Heart J. 2020;41(32):3072-9.

6. Soo RJJ, Chiew CJ, Ma S, Pung R, Lee V. Decreased influenza incidence under COVID-19 control measures. Singapore Emerg Infect Dis. 2020;26(8):1933-5.

7. Wee LE, Conceicao EP, Tan JY, Sim JXY, Venkatachalam I. Reduction in asthma admissions during the COVID-19 pandemic: consequence of public health measures in Singapore. Eur Respir J. 2021;57(4).

8. Severino P, D'Amato A, Saglietto A, D'Ascenzo F, Marini C, Schiavone $\mathrm{M}$, et al. Reduction in heart failure hospitalization rate during coronavirus disease 19 pandemic outbreak. ESC Heart Failure. 2020;7(6):4182-8.

9. Hall ME, Vaduganathan M, Khan MS, Papadimitriou L, Long RC, Hernandez GA, et al. Reductions in heart failure hospitalizations during the COVID-19 pandemic. J Card Fail. 2020;26(6):462-3.

10. Cox ZL, Lai P, Lindenfeld J. Decreases in acute heart failure hospitalizations during COVID-19. Eur J Heart Fail. 2020;22(6):10456.

11. Juan HC, Chao CM, Lai CC, Tang HJ. Decline in invasive pneumococcal disease during COVID-19 pandemic in Taiwan. J Inf Secur. 2021;82(2):282-327.

12. Colivicchi F, Di Fusco SA, Magnanti M, Cipriani M, Imperoli G. The impact of the Coronavirus Disease-2019 pandemic and Italian lockdown measures on clinical presentation and management of acute heart failure. J Card Fail. 2020;26(6):464-5.

13. Bromage DI, Cannatà A, Rind IA, Gregorio C, Piper S, Shah AM, et al. The impact of COVID-19 on heart failure hospitalization and management: report from a Heart Failure Unit in London during the peak of the pandemic. Eur J Heart Fail. 2020;22(6):978-84.

14. Jiménez-Blanco Bravo M, Cordero Pereda D, Sánchez Vega D, Del Prado DS, Monteagudo Ruiz JM, Zamorano Gómez JL, et al. Heart failure in the time of COVID-19. Cardiology. 2020;145(8):481-4.

15. Cannatà $\mathrm{A}$, Bromage DI, Rind IA, Gregorio C, Bannister C, Albarjas M, et al. Temporal trends in decompensated heart failure and outcomes during COVID-19: a multisite report from heart failure referral centres in London. Eur J Heart Fail. 2020;22(12):2219 24.

16. Barghash MH, Pinney SP. Heart failure in the COVID-19 pandemic: where has all New York's congestion gone? J Card Fail. 2020;26(6):477-8.

17. Cox ZL, Lewis CM, Lai P, Lenihan DJ. Validation of an automated electronic algorithm and "dashboard" to identify and characterize decompensated heart failure admissions across a medical center. Am Heart J. 2017;183:40-8.

Publisher's Note Springer Nature remains neutral with regard to jurisdictional claims in published maps and institutional affiliations. 